Revista peruana de biología 26(1): 003 - 008 (2019) doi: http://dx.doi.org/10.15381/rpb.v26i1.15902 ISSN-L 1561-0837; elSSN: 1727-9933
Universidad Nacional Mayor de San Marcos

\section{Una especie nueva ovo - imitadora en Passiflora (Passifloraceae) de la Provincia Huancabamba, Piura, Perú}

\author{
A new egg - mimic Passiflora species (Passifloraceae) from \\ Huancabamba Province, Piura, Peru
}

\section{TRABAJOS ORIGINALES}

$\begin{array}{ll}\text { Presentado: } & 28 / 01 / 2018 \\ \text { Aceptado: } & 22 / 12 / 2018 \\ \text { Publicado online: } & 30 / 03 / 2019\end{array}$

\section{Correspondencia:}

Universidad Nacional Pedro Ruiz Gallo, Ciudad Universitaria Juan XXIII 391, Lambayeque, Perú. Email: kamijo002@gmail.com

\section{Boris Esquerre-Ibañez}

Universidad Nacional Pedro Ruiz Gallo, Lambayeque, Perú.

\section{Citación:}

Esquerre-Ibañez. 2019. Una especie nueva ovo imitadora en Passiflora (Passifloraceae) de la Provincia Huancabamba, Piura, Perú. Revista peruana de biología 26(1): 003 - 008 (Febrero 2019). doi: http://dx.doi.org/10.15381/rpb.v26i1.15902

Palabras clave: Passiflora; Passifloraceae; Passiflora Subgénero Decaloba; Porcuya; Perú.

Keywords: Passiflora; Passifloraceae; Passiflora Subgenus Decaloba; Porcuya; Peru

\section{Resumen}

Passiflora santos-llatasii es descrita e ilustrada como una nueva especie en Passiflora subgénero Decaloba (Passifloraceae). Es endémica de los bosques montanos de neblina occidentales de la Provincia de Huancabamba, Departamento de Piura, en el noroeste del Perú. Esta especie es similar a P. pardifolia del Brasil pero se diferencian principalmente en el color de las hojas, tamaño de brácteas, corona floral tanto en color como el número de series, forma del fruto, y la distribución de sus poblaciones silvestres. Se añaden datos adicionales sobre su etimología, ecología, distribución, conservación y se discute su parentesco con otras especies afines.

\section{Abstract}

Passiflora santos-llatasii is described and illustrated as a new species in Passiflora subgenus Decaloba (Passifloraceae). It is endemic to western cloud forests from the Huancabamba Province in Piura Department, in northern Peru. This species is similar to the Brazilian P. pardifolia, but differs mainly in leaves color, bracts size, flower corona -both in color and series number, fruit shape, and distribution of wild populations. Additional information about etymology, ecology, distribution and conservation is added, with discussion of similar species.

\section{Introducción}

El género Passiflora comprende especies trepadoras herbáceas a leñosas, arbustos y arbolitos (Gentry 1996), tiene importancia comercial por sus frutos comestibles, así como por su potencial ornamental y sus principios activos (Hernández \& Bernal 2000; Dhawan et al. 2004), y además, su adaptación morfológica y relaciones planta-animal son de interés en estudios ecológicos y evolutivos (Gilbert 1982, Büchert \& Mogens 2001, Muchhala \& Jarrin 2002, Leal et al. 2006).

En el Perú, Brako \& Zarucchi (1993) y Ulloa et al. (2004) reportaron un total de 95 especies para el género Passiflora; pero, en los últimos años se han ido adicionando nuevos taxones (Boza 2014, Leiva \& Tantaleán 2015, 2016). Durante la revisión del género Passiflora en el norte del Perú, que empezó como una iniciativa propia del autor, hace más de 5 años, se ha venido reportando y publicando nueva información, nuevos registros y nuevas especies (Esquerre et al. 2014, Esquerre 2015a, 2015b), y recientemente en el marco del proyecto 
"Estudios taxonómicos selectos en la flora del Norte del Perú" se ha continuado la labor científica de seguir investigando este interesante género (Esquerre 2017), y por ese motivo se presenta aquí la descripción y detalles adicionales de una especie nueva para el Perú, un nuevo endemismo de la provincia de Huancabamba (Piura) y un acierto más para promover la conservación in situ y ex situ de especies de zonas biológicamente importantes, pero que no están protegidas por el Estado peruano.

\section{Passiflora santos-llatasii B. Esquerre sp. nov.}

$$
\text { (Figs. 1 - 4) }
$$

In affinity with Passiflora pardifolia Vanderplank, differs mainly in possessing variegated leaves with adaxial white - reddish strips, flowers with white-green biseriate corona, ovoid oblong ripe fruit with the lower end acute.

TIPO: PERÚ, Departamento de Piura, provincia de Huancabamba, distrito de Huarmaca, Limón de Porcuya, 1560 m. 552'58'S-79³2'13'”, 17 junio 2017, Esquerre 163 (holotipo HUT!; isotipos HUT!, CPUN!).

Trepadora herbácea, semileñosa cuando longeva, con tricomas de $0.2-0.3 \mathrm{~mm}$, curvados generalmente en toda la planta. Tallo estriado, casi aplanado en plantas maduras. Estípulas falcadas, 4 - 5 x 0.7 - $1 \mathrm{~mm}$, verdosas a púrpuras. Peciolo $1-5 \mathrm{~cm}$ de largo, angulado, estriado, sin glándulas; lámina 2 - lobada, $1-5.2$ × $2-11 \mathrm{~cm}$, raramente con un lobo inconspicuo central, nervaduras laterales de hasta $10 \mathrm{~cm}$ de largo, margen entero, cara adaxial verde o variegada con máculas blancas, rosas a rojizas sobre las nervaduras laterales y a veces la nervadura central, sección entre las nervaduras laterales con 4 - 10 glándulas laminares, amarillentas o a veces rosadas, y con relieve en la cara adaxial, redondeadas, marrónamarillas y nectaríferas en la cara abaxial, desde la base hacia el ápice, siguiendo las máculas. Brácteas 3, libres, enteras, intercaladas, linear-lanceoladas, $3-4$ x $0.4-$ $0.6 \mathrm{~mm}$. Botones florales de hasta 1.8 x $2.5 \mathrm{~cm}$. Flores en antesis de $5-5.5 \mathrm{~cm}$ de diámetro, en pares; hipantio corto campanulado, de hasta $6 \mathrm{~mm}$ de altura; sépalos 5 oblongo-lanceolados, ensanchados en la base, verdosos con matices rojizos a violáceos abaxialmente, blancos adaxialmente, $1.8-2 \times 0.4-0.6 \mathrm{~cm}$; pétalos 5 , oblongo-lanceolados, blancos, $1.5-1.6 \times 0.4-0.5 \mathrm{~cm}$. Corona filamentosa en 2 series, raramente 3 , serie más externa de hasta $18 \mathrm{~mm}$ de largo, blanca a crema, terete, serie interna de filamentos casi aplanados con ápice irregular con protuberancias, verde, $4-7 \mathrm{~mm}$ de largo, filamentos libres o a veces fusionados en la base o hasta la mitad, a veces suele aparecer una tercera serie derivada de estos filamentos verdosos. Opérculo plicado, verdoso, rosado y blanquecino, de margen irregular, $5 \mathrm{~mm}$ de altura. Limen de 1 - $1.5 \mathrm{~mm}$, incurvado hacia el androginóforo, blanquecino, con máculas moradas dispersas. Androginóforo de hasta $8 \mathrm{~mm}$ de altura. Anillo nectarífero inconspicuo. Tróclea ausente. Filamentos estaminales 5 - 6 mm, verdosos a púrpuras, anteras $3 \times 5 \mathrm{~mm}$, amarillas a verdosas. Ovario pubescente $7 \times 4 \mathrm{~mm}$, verdoso, estilos 3 , de hasta $8 \mathrm{~mm}$ de largo, verdosos con máculas púrpuras hacia el estigma, estigma crema a amarillento. Fruto baya ovoide - oblongo, inmaduro verde, maduro negruzco, con la parte inferior mamilada (aguda), de hasta 4 x $2.5 \mathrm{~cm}$. Semillas de hasta $4.5 \times 3 \mathrm{~mm}$, con un cuerno apical, borde entero, costadas transversalmente con $5-6$ surcos, base aguda curvada, negras, envueltas en un arilo translúcido a amarillento.

\section{Discusión}

Passiflora santos-llatasii guarda especial afinidad con P. pardifolia, especialmente en las hojas bilobadas que se parecen mucho por la forma del limbo, la variegación y la presencia de ocelos amarillentos en la cara adaxial, pero también hay diferencias, información que se resume en la Tabla 1, principalmente vinculadas al tamaño de las estructuras florales como la corona.

Se propone la ubicación de esta nueva especie del subgénero Decaloba en la supersección Decaloba sección Decaloba DC. de acuerdo a la actual clasificación infragenérica de Passiflora (Feuillet \& MacDougal 2004) por poseer opérculo plicado, ápice de rama cernuo y presencia

Tabla 1. Diferencias morfológicas notables entre Passiflora santos - Ilatasii y P. pardifolia

\begin{tabular}{|c|c|c|}
\hline Características & Passiflora pardifolia & Passiflora santos - Ilatasii \\
\hline Hojas & $\begin{array}{l}\text { Bilobadas con variegación blanquecina adaxial y ocelos } \\
\text { amarillentos }\end{array}$ & $\begin{array}{l}\text { Bilobadas con variegación blanquecina - rojiza adaxial y ocelos } \\
\text { amarillentos }\end{array}$ \\
\hline Brácteas & $2-2.5 \mathrm{~mm}$ de largo & $3-4 \mathrm{~mm}$ de largo \\
\hline Flores & $4-4.4 \mathrm{~cm}$ de diámetro & $5-5.5 \mathrm{~cm}$ de diámetro \\
\hline Sépalos & $14-17 \times 5-7 \mathrm{~mm}$ & $18-20 \times 4-6 \mathrm{~mm}$ \\
\hline Pétalos & $9-11 \times 4-4.5 \mathrm{~mm}$ & $15-16 \times 4-5 \mathrm{~mm}$ \\
\hline \multirow{2}{*}{ Corona } & $\begin{array}{l}\text { Serie externa de hasta } 16 \mathrm{~mm} \text { de largo. Blanca con } 1-2 \\
\text { bandas malva pálido }\end{array}$ & Serie externa de hasta $18 \mathrm{~mm}$ de largo. Blanca sin bandas \\
\hline & Serie interna filiforme, blanca, de hasta $3 \mathrm{~mm}$ de largo & $\begin{array}{l}\text { Serie interna casi aplanada de ápice irregular, verdosa, de hasta } \\
7 \mathrm{~mm} \text { de largo }\end{array}$ \\
\hline Operculum & Verde pálido & Verde intenso con máculas púrpura \\
\hline Estilos & Púrpura profundo de hasta $6 \mathrm{~mm}$ de largo & Verdes con máculas púrpuras de hasta $8 \mathrm{~mm}$ de largo \\
\hline Fruto & Baya negra globosa a subglobosa, parte inferior redondeada & Baya negra ovoide - oblonga, parte inferior aguda mamilada \\
\hline Semillas & Marrón oscuro de hasta 4.1 × $2.5 \mathrm{~mm}$ & Negras de hasta $4.5 \times 3 \mathrm{~mm}$ \\
\hline
\end{tabular}



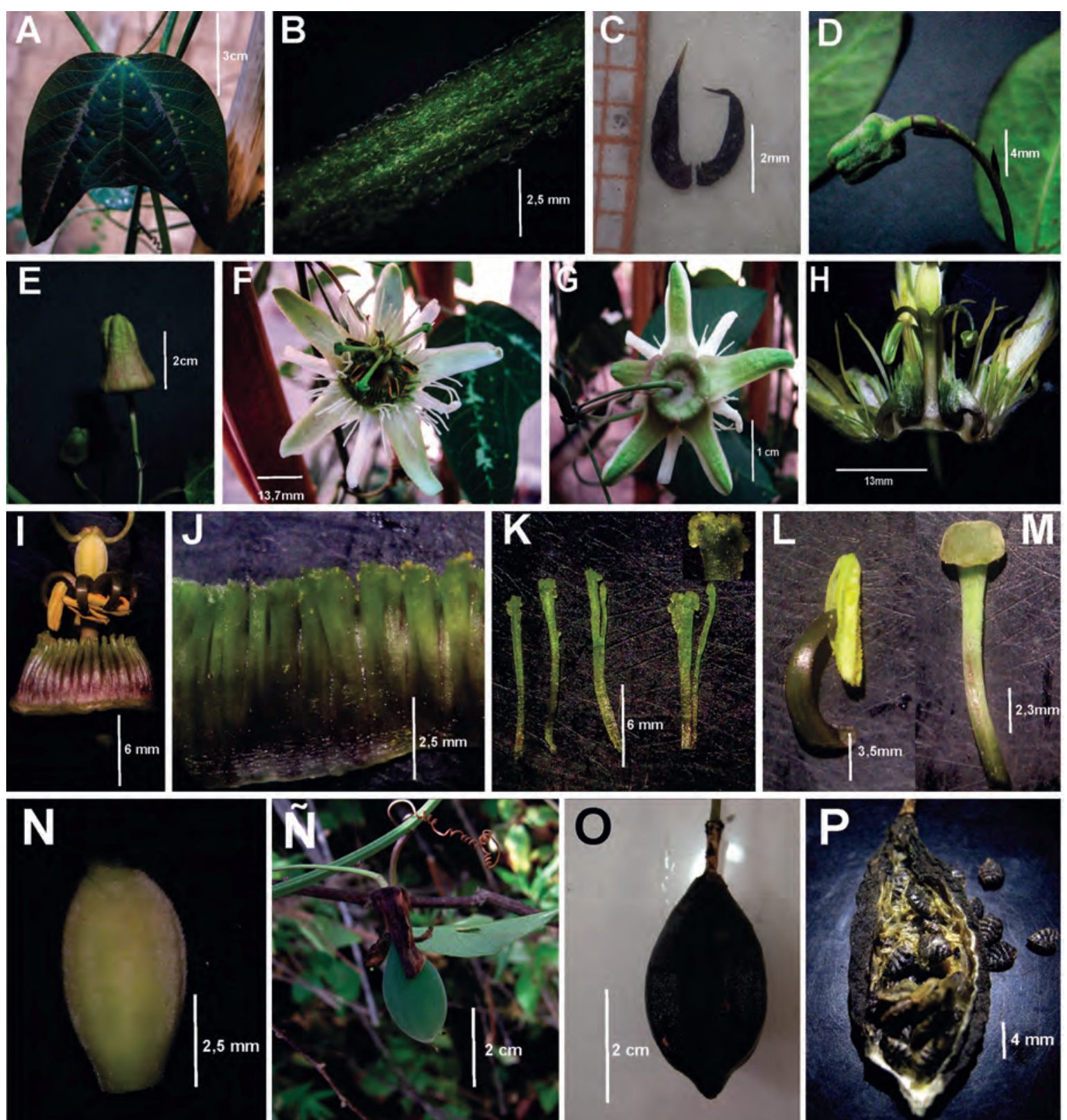

Figura 1. Passiflora santos - Ilatasii B. Esquerre sp. nov. : (A) Lámina foliar, (B) Tallo, (C) Estípulas, (D) Brácteas, (E) Botón floral, (F) Flor en antesis: cara adaxial, (G) Flor en antesis: cara abaxial, (H) Transcorte de flor, (I) Androginóforo y opérculum, (J) Opérculum, (K) Filamentos internos, (L) Estambre, (M) Estilo, (N) Ovario, (N) Fruto inmaduro, (O) Fruto maduro, (P) Semillas. (Esquerre 163)

de nectarios laminares. La sección Decaloba DC., importante y numeroso linaje de la monofilética supersección Decaloba (Krosnick et al. 2013) está presente en el Perú en casi todo el territorio nacional con aproximadamente 16 especies, habitando especialmente la ceja de selva occidental, oriental y los llanos amazónicos.

Dentro de este grupo, otras especies afines en el hábito y las hojas lo son Passiflora indecora Kunth y P. punctata Linnaeus, habitantes también de la zona noroeste montana del Perú, estando presente la primera generalmente sobre los $2000 \mathrm{~m}$ a diferencia de la segunda de zonas más bajas, e incluso costeras. Especies fuera del rango geográfico nacional que también se parecen por el hábito foliar a P. santos-llatasii lo son P. boenderi MacDougal de Costa Rica y P. popayanensis Killip de Colombia por ejemplo. Cabe mencionar que todas estas especies tienen flores completamente diferentes a la nueva especie.

Es interesante la presencia de variegación rosada a rojiza sobre las nervaduras laterales principales en la cara adaxial del limbo de las hojas de esta nueva especie, característica rara observada por ejemplo también en Passiflora trifasciata Lemaire, la brasileña P. organensis Gardner y la ecuatoriana P. telesiphe Knapp \& Mallet, detalle que se puede aprovechar en este grupo de especies para un uso ornamental de las plantas, vinculado a la conservación ex situ de las mismas.

Especies semejantes en flor, a $P$. santos-llatasii por ejemplo lo son $P$. urnifolia Rusby y la todavía inédita $P$. cana (J. MacDougal com. pers.) especialmente en el operculum verdoso y la corona externa blanca. 
Especímenes adicionales examinados. - Passiflora santos-llatasii: PERÚ. Departamento de Piura, provincia de Huancabamba, distrito de Canchaque. Arriba de Canchaque. 1400 - 1500 m. 22 marzo 1948. R. Ferreyra 3111 (MO! imagen). Chorro Blanco. 1500 - 1900 m. 18 abril 1987. C. Díaz \& S. Baldeón 2467 (MO! imagen). Distrito de Huarmaca. Carretera de Olmos a Tambo. $1750 \mathrm{~m}$. 23 mayo 1998. M. Weigend \& N. Dostert 98/295 (USM!).

Registros adicionales in situ sin colecta. - Passiflora santos-llatasii: PERÚ. Departamento de Piura, provincia de Huancabamba, distrito de Huarmaca. Entrada a Limón de Porcuya, 1500 m, 12 noviembre 2012. Quebrada antes de Limón, 1569 m, 553'5"S-79³2'4"W, 31 de enero 2015. Ruta hacia La Coipa, $2363 \mathrm{~m}, 5^{\circ} 50^{\prime} 6^{\prime \prime} \mathrm{S}$ -79³1'8'W, 12 de junio 2015. Quebrada antes de Limón, $1541 \mathrm{~m}, 5^{\circ} 53^{\prime} 6.1^{\prime \prime} \mathrm{S}-79^{\circ} 32^{\prime} 5.1^{\prime \prime} \mathrm{W}, 9$ de julio 2016. Distrito de Canchaque. sobre Palambla, $1126 \mathrm{~m}, 5^{\circ} 23^{\prime} 13^{\prime \prime S}$ 79³6'31"W, 03 de febrero 2018.

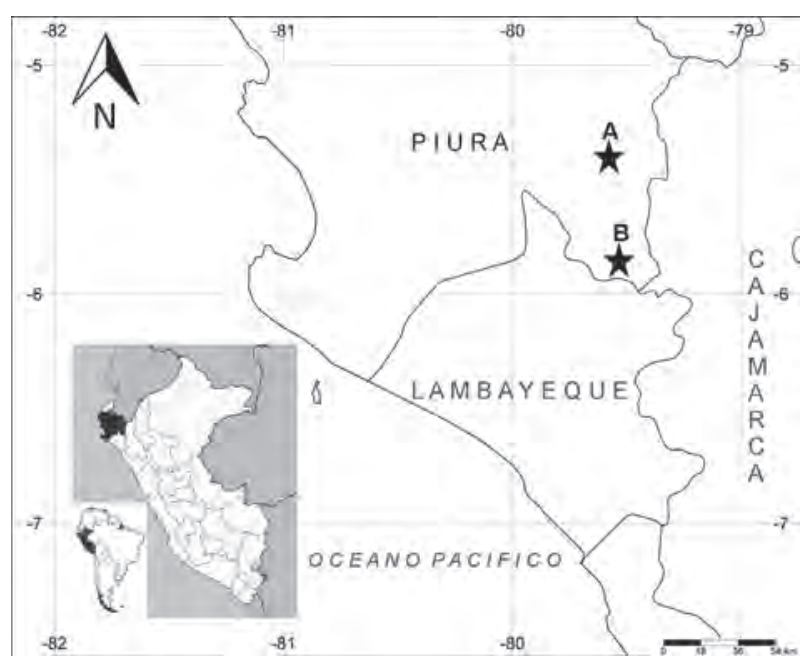

Figura 2. Distribución preliminar de las poblaciones de Passiflora santos-llatasii en el Departamento de Piura, Provincia Huancabamba: (A) Distrito Canchaque, (B) Distrito Huarmaca.

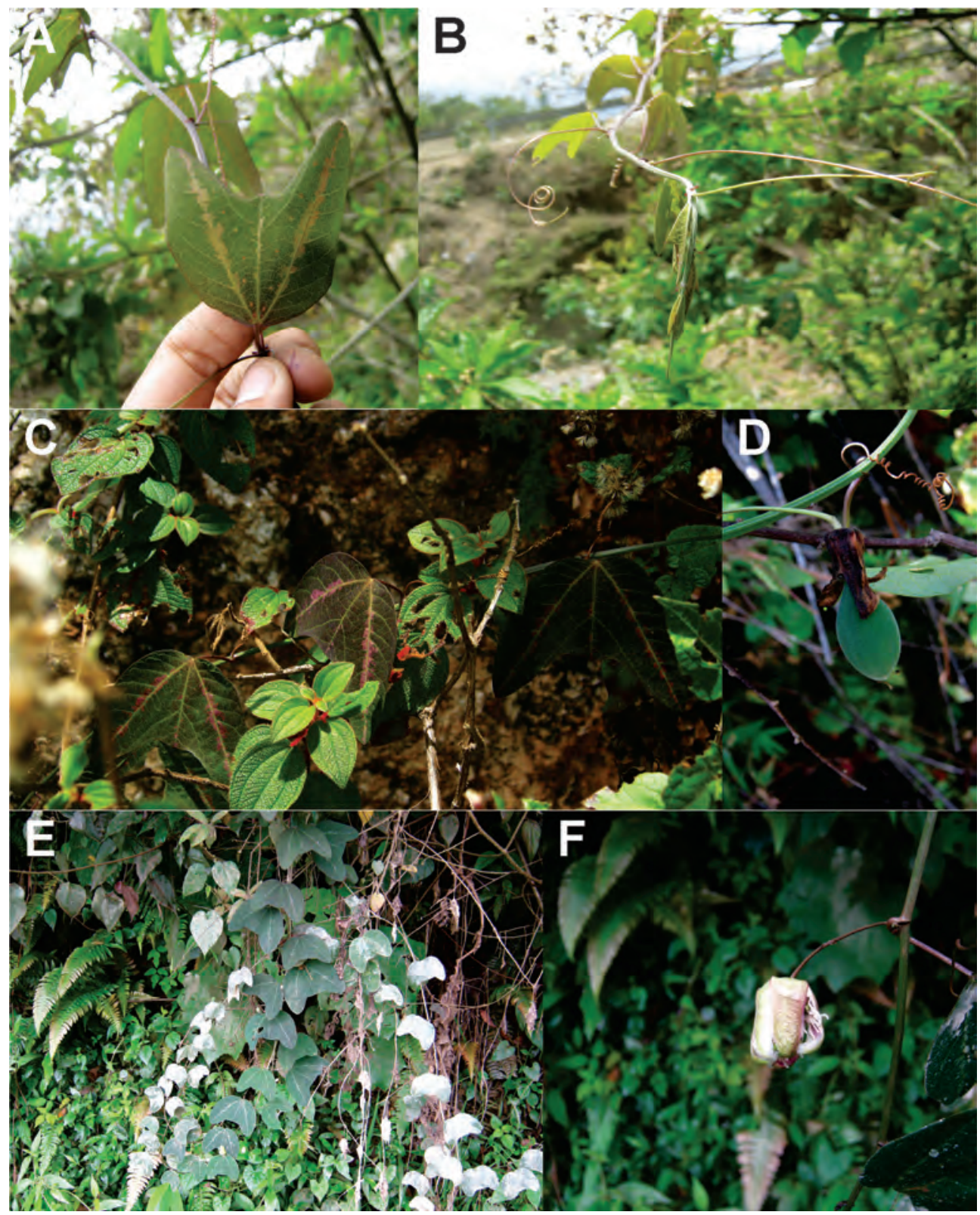

Figura 3. Passiflora santos-Ilatasii en su hábitat: Distrito Huarmaca (A-B) Limón de Porcuya, (C-D) Ruta a La Coipa. Distrito Canchaque (E-F) Palambla. 


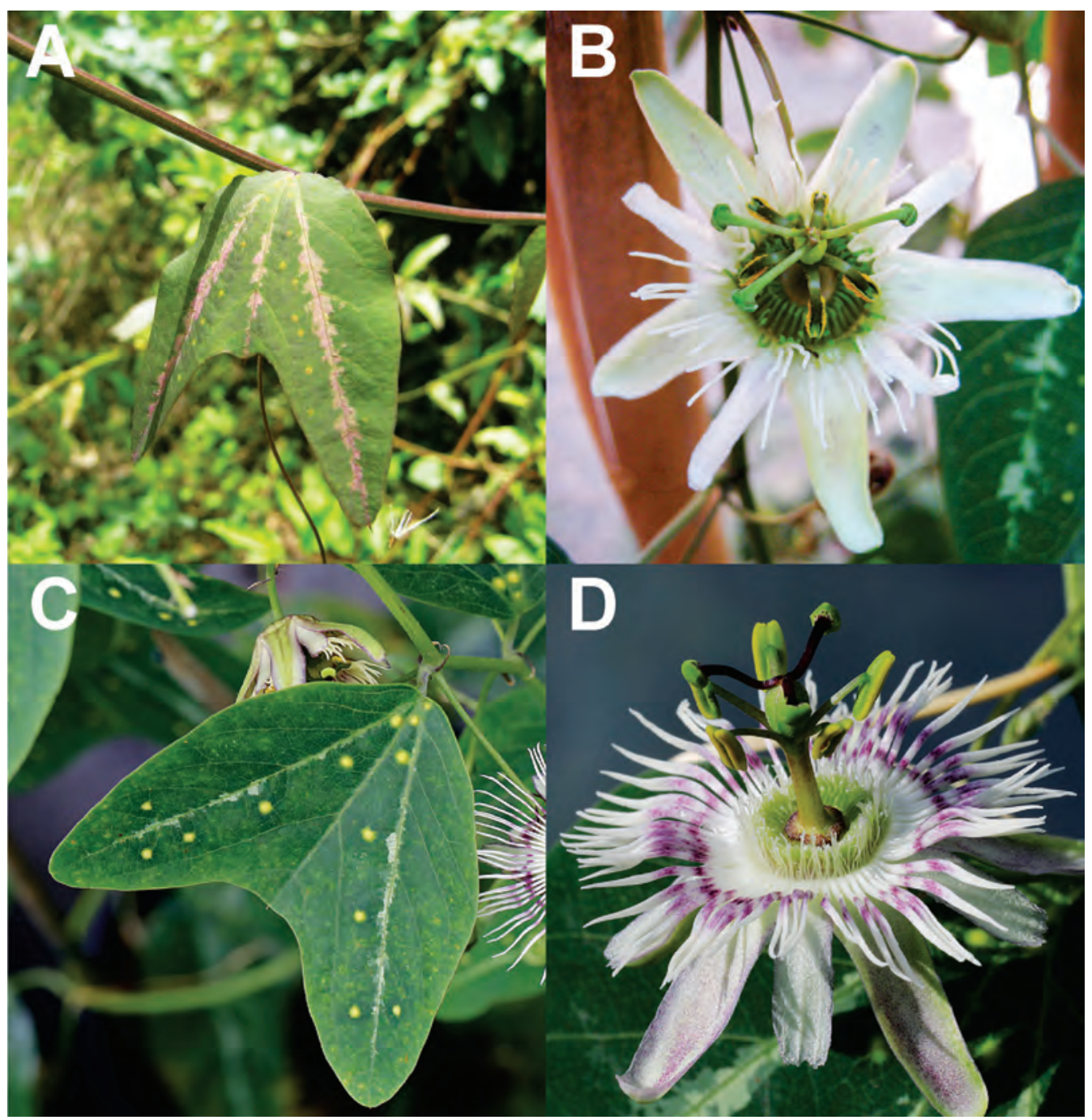

Figura 4. Passiflora santos-Ilatasii: (A) Hoja, (B) Flor. Passiflora pardifolia: (C) Hoja, (D) Flor. (P. pardifolia C-D fotografías de Maurizio Vecchia)

Distribución y estado de conservación.- Passiflora santos-llatasii ha sido colectada y vista solamente en la provincia de Huancabamba, en los distritos de Canchaque y Huarmaca, en hábitat de bosque montano desde los 1100 hasta los $2300 \mathrm{~m}$ a diferencia de su afín P. pardifolia que habita en el Estado de Minas Gerais, Brasil. Constituye ahora un nuevo endemismo para la flora peruana y se propone su clasificación de acuerdo a las categorías de la UICN (IUCN 2013) como En Peligro: EN B1ab (iii), al no haber encontrado más poblaciones en los alrededores, hasta que quizás nuevos reportes amplíen su distribución a localidades vecinas, por lo que mientras tanto sus poblaciones están desprotegidas y amenazadas por la agricultura migratoria y la expansión urbana de zonas rurales.

Ecología-- La presencia de ocelos amarillentos es muy evidente en el limbo, haciéndose más notorios cuando la hoja toma una coloración verde oscuro y violácea, por la presencia de antocianinas, pudiendo existir una relación planta-animal de mucha importancia en el entorno de su área de distribución, probablemente con los miembros de la subfamilia de mariposas Heliconiinae
(Lepidoptera: Nymphalidae), donde los ocelos cumplirían una señalización de puesta de huevos de las hembras, pudiendo esto evitar en parte la ovoposición de hembras que buscan asiduamente las hojas de Passiflora y por ende su infestación con las futuras voraces larvas (Gilbert 1982), así como algunas especies conocidas donde también se ha discutido la mímica foliar de huevos de mariposa, por ejemplo Passiflora boenderi, P. pardifolia, P. gilbertiana MacDougal, P. mexicana Jussieu, P. ornithou$r a$ Masters, entre otras especies de la sección Decaloba, grupo donde prevalece esta mimetización (MacDougal 2003, Vanderplank 2006).

Durante el período no lluvioso en su hábitat, $P$. santosllatasii pierde generalmente todas sus hojas permaneciendo reducida a bejucos casi secos, muchas veces enterrados bajo la hojarasca del bosque, donde esperan las próximas precipitaciones para empezar a rebrotar y hacerse notar al trepar por las ramas de sus especies vecinas.

Etimología. - Passiflora santos-llatasii en honor a Santos Régulo Llatas Quiroz, quien en vida fue botánico, colector y profesor del autor, como homenaje póstumo 
a su labor científica en la flora local de Lambayeque, del norte del Perú y su colaboración con los herbarios del extranjero, acción gracias a la cual se ha llevado a cabo el descubrimiento de muchas especies nuevas.

\section{Literatura citada}

Boza T. 2004. Two new species of Passiflora subg. Decaloba (Passifloraceae) from Peru and Bolivia. Novon 23(3):263 - 267. http://doi.org/10.3417/2012066

Brako L \& J. Zarucchi. 1993. Catálogo de plantas con flores y gimnospermas del Perú. Monographs in Systematic Botany 45. Missouri Botanical Garden, St. Louis, MO.

Büchert A \& J. Mogens. 2001. The fragility of extreme specialization: Passiflora mixta and its pollinating hummingbird Ensifera ensifera. J. Trop. Ecol. 17: 323 - 329. http://dx.doi.org/10.1017/S0266467401001213

Dhawan K; S. Dhawan \& A. Sharma. 2004. Passiflora: a review update. J. Ethnopharmacol. 94: 1 - 23. http://dx.doi. org/10.1016/j.jep.2004.02.023

Esquerre - Ibañez B. 2015a. A new species of Passiflora supersection Tacsonia (Passifloraceae) from Amazonas, Northern Peru. Phytotaxa 202(4): 266 - 272. http:// dx.doi.org/10.11646/phytotaxa.202.4.4

Esquerre - Ibañez B. 2015b. Passiflora dorisiae, una nueva especie en el subgénero Passiflora (Passifloraceae). Revista Peruana de Biología 22(3): 303 - 308. http:// dx.doi.org/10.15381/rpb.v22i3.11435

Esquerre - Ibañez B. 2017. A new species of Passiflora section Granadillastrum (Passifloraceae) from Jaén, Peru. Phytotaxa 298(1): 89 - 95. http://doi.org/10.11646/ phytotaxa.298.1.10

Esquerre - Ibañez B.; C. Rojas - Idrogo, S. Llatas - Quiroz \& G. Delgado - Paredes. 2014. El género Passiflora L. (Passifloraceae) en el Departamento de Lambayeque, Perú. Act. Bot. Malac. 39: 55 - 70.

Feuillet C. \& J. M. MacDougal. 2004. A new infrageneric classification of Passiflora. Passiflora 13(2): 34 - 35, 37 - 38.

Gentry A. K. 1996. A field guide to the families and genera of woody plants of northwest South America (Colombia, Ecuador, Peru) with supplementary notes on herbaceous taxa. University of Chicago Press, Chicago. 918 pp.

Gilbert L. 1982. The coevolution of a butterfly and a vine. Sci. Am. 247: 110 - 121. http://dx.doi.org/10.1038/ scientificamerican0882- 110

Hernández A. \& R. Bernal. 2000. Lista de Especies de Passifloraceae de Colombia. Biota Colombiana 1(3): 320 - 335.

IUCN. 2013. Guideline for using the IUCN Red List Categories and Criteria. Version 10. Prepared by the Standards and Petitions Subcommittee. Downloadable from http://www.iucnredlist.org/documents/RedListGuidelines.pdf.
Krosnick S.; K. Porter - Utley, J. MacDougal, P. Jørgensen \& L. MacDade. 2013. New insights into the evolution of Passiflora Subgenus Decaloba (Passifloraceae): Phylogenetic relationships and morphological synapomorphies. Systematic Botany 38(3): 692 - 713. https:// doi.org/10.1600/036364413X670359

Leal I.; E. Fischer, C. Kost, M. Tabarelli \& R. Wirth. 2006. Ant protection against herbivores and nectar thieves in Passiflora coccinea flowers. Ecoscience 13 (4): 431 438. http://dx.doi.org/10.2980/11956860(2006)13[ 431:APAHAN]2.0.CO;2

Leiva S. \& Tantaleán F. 2015. Passiflora salpoense (Passifloraceae) una nueva especie del Norte del Perú. Arnaldoa 22(1): $35-48$.

Leiva S. \& Tantaleán F. 2016. Lectotipificación de Passiflora salpoense (Passifloraceae). Arnaldoa 23(2): 627 - 630. http://doi.org/10.22497/arnaldoa.232.23213

MacDougal J. 2003. Passiflora boenderi (Passifloraceae), a new egg - mimic passionflower from Costa Rica. Novon 13(4): 454 - 457. http://doi.org/10.2307/3393379

Muchhala N. \& P. Jarrin - V. 2002. Flower visitation by bats in cloud forests of western Ecuador. Biotropica 34: 387 - 395. http://dx.doi.org/10.1646/00063606(2002)0 34[0387:FVBBIC]2.0.CO;2

Ulloa C.; J. Zarucchi \& B. León. 2004. Diez años de adiciones a la Flora del Perú: 1993 - 2003. Arnaldoa (edición especial): 7 - 242. http://dx.doi.org/10.5962/bhl.title.63538

Vanderplank J. 2006. 565. Passiflora pardifolia. Passifloraceae. Curtis's Botanical Magazine 23(3): 243 - 247. http:// doi.org/10.1111/j.1467 - 8748.2006.00536.x

\section{Agradecimientos:}

A Santos Llatas por ser la primera persona que comentó al autor acerca de esta especie rara en las proximidades de la localidad tipo; John MacDougal por su valiosa ayuda en la determinación taxonómica y ubicación de paratipos, Consuelo Rojas, Josefa Escurra y Leopoldo Vásquez por facilitar la movilidad numerosas veces en la ruta hacia el Abra Porcuya. A Guillermo Delgado por permitir el estudio morfológico con uso del ambiente y equipos del Laboratorio de Cultivo de Tejidos Vegetales y Recursos Fitogenéticos de la Universidad Nacional Pedro Ruiz Gallo en Lambayeque, Perú. A Rodrigo Rijalba por su colaboración en las colectas de campo. A los curadores de los herbarios HUT y CPUN por permitir el depósito de especímenes tipo en sus herbarios. A Yero Kuethe, Myles Irvine y Maurizio Vecchia por su colaboración con material fotográfico de Passiflora pardifolia.

\section{Aspectos éticos / legales:}

SERFOR mediante Resolución N² 247 - 2016 - SERFOR/DGGSPFFS. Este trabajo no incurrió en ningun problema ético.

\section{Conflicto de intereses:}

Los autores no incurren en conflictos de intereses.

\section{Fuentes de financiamiento:}

El presente trabajo no contó con una fuente de financiamiento específica. 